\title{
A neonate born to an infected COVID-19 mother was tested positive just 24 hours after its birth.
}

Roya Huseynova $^{1}$, Latifa A.Bin Mahmoud ${ }^{1}$, Adli Abdelrahim ${ }^{1}$, Mishary Almalkey ${ }^{1}$, Oqtay Huseynov $^{2}$, Haider Sumaily ${ }^{1}$, and Ameen Amer Almotiri ${ }^{1}$

${ }^{1}$ King Saud Medical City

${ }^{2}$ Azerbaijan Medical University Nariman Narimanov

July 22, 2020

\begin{abstract}
Coronavirus disease (COVID-19) is a highly contagious disease with a rapid increase in cases and mortality all over the world. We report a neonate born to the pregnant woman with COVID tested positive for SARS-CoV-2 infection just 24 hours after its birth.
\end{abstract}

A neonate born to an infected COVID-19 mother was tested positive just 24 hours after its birth.

Roya Arif Huseynova ${ }^{1}$, Latifa A.Bin Mahmoud ${ }^{1}$, Adli Abdelrahim ${ }^{1}$, Mishary Almalkey ${ }^{2}$, Oqtay Huseynov ${ }^{3}$ , Haider Hussein Sumaily ${ }^{1}$, Ameen Amer Almotiri ${ }^{1}$

${ }^{1}$ Neonatology Department, King Saud Medical City, Riyadh, Saudi Arabia

${ }^{2}$ Pediatric Department, King Saud Medical City, Riyadh, Saudi Arabia

${ }^{3}$ Azerbaijan Medical University, Baku, Azerbaijan

Corresponding author: Roya Arif Huseynova,

Neonatal Intensive Care Unit, King Saud Medical City, Al Imam Abdul Aziz Ibn Muhammad Ibn Saud 12746, Riyadh, Kingdom of Saudi Arabia.

E-mail: huseynova_roya@yahoo.com,r.huseynova@ksmc.med.sa

Telefon: 00966508463068

ORCiD id 0000-0002-8914-5892

A neonate born to an infected COVID-19 mother was tested positive just 24 hours after its birth.

Keywords: COVID-19, newborn, coronavirus, SARS-CoV-2, infection

\section{Key Clinical Message}

Coronavirus disease (COVID-19) is a contagious disease with a rapid increase in cases and mortality all over the world. We report a newborn of a pregnant woman with COVID-19, who was confirmed positive for COVID-19 by (rt-PCR) after a nasopharyngeal swab was taken at 24 hours of age.

Introduction 
Coronavirus disease (COVID-19) is caused by severe acute respiratory syndrome coronavirus 2 (SARS-CoV2) was first detected in the city of Wuhan, the capital of Hubei Province, China, in December $2019^{1}$.

Several articles report the outcomes of pregnant women infected in the present course of COVID-19 pandemic. However, established reports and findings of intrauterine infection attributed to vertical transmission in infected pregnant women with SARS-CoV-2 is limited.

\section{Case report}

We report a 37 weeks' gestation newborn who was confirmed by real-time-reverse-transcription polymerase chain reaction (rt-PCR) to be positive after a nasopharyngeal swab was taken at 24 hours of age.

The mother of the index case is a 16-year-old pregnant woman who presented to the maternal emergency department on June 13th, 2020, at 17:0 due to premature rupture of membrane (PROM). She was a primigravida, and her previous medical history was unremarkable of any medical or pregnancy-related illnesses. She was following up on her pregnancy in a private hospital. There was no history of suspected or confirmed individuals with COVID-19 among her family members.

Her initial assessment was body temperature $36.5^{\circ} \mathrm{C}$, blood pressure $112 / 66 \mathrm{~mm} \mathrm{Hg}$, respiratory rate 20 breaths per minute, heart rate 118 beats per minute, and oxygen saturation $95 \%$ in room air. She didn't have any upper respiratory symptoms (cough, sneezing, or sputum). Fetal heart monitoring was reassuring between 130-140 beats per minute, with no signs of abnormality.

The mother was admitted to the prenatal ward for observation and started on intravenous ceftriaxone because of premature rupture of membrane and amniotic fluid leakage.

Blood tests showed white blood cells count 10.8 x109 cells /L, neutrophil 7.67x109 cells/L, hemoglobin level 10 $\mathrm{g} / \mathrm{dl}$, platelet count 194 x109 cells /L; coagulation profile (prothrombin time $11.8 \mathrm{sec}$, partial thromboplastin time $28.7 \mathrm{sec}$, international normalized ratio1.1; erythrocyte sedimentation rate $6 \mathrm{~mm} / \mathrm{h}$, liver function tests: aspartate aminotransferase $22.6 \mathrm{U} / \mathrm{L}$, alanine transaminase $9.8 \mathrm{U} / \mathrm{L}$ and ferritin level was $74.5 \mathrm{ng} / \mathrm{ml}$.

At 00:20 on June 14th, 2020, the mother developed chills, felt feverish, and was complaining of back pain. Her body temperature was $37.8^{\circ} \mathrm{C}$. She was given oral paracetamol for fever. She was suspected of having COVID-19; thus, a nasopharyngeal swab for COVID-19 was taken at 04:30 the same day as per hospital policy. At 6:00, she continued to have fever $37.8^{\circ} \mathrm{C}$ as well as mild dry cough and relative tachycardia. Her chest x-ray was unremarkable. Fortunately, at 8:30, her body temperature and tachycardia subsided, and fetal heart monitoring remained reassuring. Since the mother was suspected of having COVID-19, universal surgical masking and regular prevention measures for COVID-19 were practiced by healthcare workers who attended the delivery room.

Following the local guidelines, the delivery took place in the isolation room. A decision was made not to allow the skin to skin contact between the baby and her mother, neither breastfeeding.

The baby girl was delivered on June 14th at 17:00 via vaginal delivery without any complications. She was immediately shifted to a separate room where initial steps of resuscitation were applied. Apgar scores were 8, 9, and 9 at 1, 5, and 10 minutes respectively. Physical examination revealed a well-appearing baby.

Birth weight was 3070 grams (50th to 90 th percentile), length of $52 \mathrm{~cm}$ (50th to 90th percentile), and her head circumference of $35 \mathrm{~cm}$ (50th percentile).

The newborn was admitted to the level-II neonatal intensive care unit (NICU) in an isolation room with a continuous cardiac monitor. She was afebrile, not distressed, and maintained her saturation above $95 \%$ on room air.

On June 14th, at 20.00 nasopharyngeal swab taken from mother came positive for COVID -19.

Nasopharyngeal swab sampling for the baby was taken at the age of 24 hours and also came positive. 
Her laboratory blood tests showed white blood cells 14.27 x109 cells /L, neutrophil 9.39 x109 cells/L, hemoglobin $17.4 \mathrm{~g} / \mathrm{dl}$, platelet $231 \times 109 / \mathrm{L}$ and hematocrit $57 \%$; C-reactive protein 3.11 (positive $>10 \mathrm{mg} / \mathrm{L}$ ); blood gas analysis $\mathrm{pH} 7.4$ with base excess -5.5 ; liver function tests: aspartate aminotransferase $16 \mathrm{U} / \mathrm{L}$ and alanine transaminase $2.9 \mathrm{U} / \mathrm{L}$. Blood culture was negative. Chest radiography didn't reveal any abnormality.

The baby continued to be asymptomatic and maintained normal vital signs in room air. She was tolerating regular formula feeding orally. Repeated nasopharyngeal swab done at 48 hours of life was negative. Both baby and mother were discharged home on day 5 with no complication. The baby was monitored until day 28 of life, and she remained asymptomatic.

\section{Discussion}

The index case is a term newborn baby with nasopharyngeal swab sample testing positive by reversetranscription polymerase chain reaction (rRT-PCR) for SARS -CoV-2 infection at 24 hours after birth. During the whole hospital course, the patient was stable.

Several studies in newborn infants are currently available, suggesting that infants usually have mild manifestations of the disease, which were consistent with our case report ${ }^{2-4}$.

However, a reported clinical analysis proposed that infants with SARS -CoV-2 infection may present with premature delivery, respiratory distress, gastrointestinal symptoms, laboratory results abnormalities like elevated liver enzymes, thrombocytopenia, and increased mortality rate ${ }^{5}$.

One of the primary risk factors of COVID-19 infection is considered close contact with an infected person within 14 days of symptoms onset and an incubation period ranging

between 2 to 14 days, an average of five days. Presently, the possibility of vertical transmission is a big question to neonatologists and obstetricians. There is limited evidence for mother-to-child transmission in women who developed SARS -CoV-2 in late pregnancy.

However, Wang et al. recently described cases of newborn infants with proven COVID-19 infection where the nasopharyngeal swabs were taken 36 hours after birth ${ }^{4}$.

A recently published cohort study identified three newborn infants with COVID-19. All these cases were delivered by the emergency cesarean section because of fetal distress and had confirmed maternal SARS -CoV-2 infection. All neonates had nasopharyngeal and anal swab positive for COVID-19 on day 2 of life despite strict infection prevention and control during procedures that were done throughout the delivery ${ }^{6}$.

Also, Lan Dong et al. reported a newborn baby with elevated anti-SARS-CoV-2 IGM antibodies two hours after the birth, and there was no physical contact with the COVID-19 positive

mother ${ }^{7}$. Detection of IgM is suggestive of the intrauterine infection. Interestingly, at the same time several requested nasopharyngeal swabs for COVID-19 were negative in the same baby.

All these neonates were isolated from the mother from the beginning, including our case, suggesting the possibility of vertical transmission in these reported cases. There is also other evidence supporting the ability of vertical transmission of SARS-CoV-2.

The latest reports identified the presence of SARS-CoV-2 in the villous chorion and amniotic fluid ${ }^{8}$. The possibility of vertical transmission can occur during vaginal birth with exposure to maternal stool infected with SARS-CoV-2 ${ }^{9}$. Additionally, SARS-CoV-2 nucleic acid was found in the blood samples ${ }^{10}$.

Unfortunately, no testing of intrauterine tissue samples as placenta, cord blood, amniotic fluid, and maternal milk was done in the index case to confirm that the SARS -CoV-2 infection occurred due to vertical transmission.

\section{Conclusion}


In order to exclude or support the possibility of congenital COVID-19, specimens from amniotic fluid, placental tissue, umbilical cord blood, gastric fluid, and maternal milk need to be additionally collected and analyzed in each neonate proven to be COVID-19 positive.

More evidence is needed before considering vertical transmission and the possibility of COVID-19 being a congenital infection.

\section{Conflict of interest}

None declared

Informed consent was obtained from parents for reporting this case.

No financial support or grant provided by any institute

\section{Author Contribution}

Roya Arif Huseynova: wrote the clinical report, collected and analyzed the data.

Latifa A.Bin Mahmoud participated in the drafting and critically revising the manuscript.

Adli Abdelrahim contributed in critically revising the manuscript.

Mishary Almalkey participated in the collected and analyzed the data.

Oqtay Ilham Huseynov participated in the drafting and collected the data.

Haider Hussein Sumaily participated in critically revising the manuscript.

Ameen Amer Almotiri participated in the collected the data.

All authors approved the manuscript as submitted.

\section{References}

1.Rasmussen SA, Smulian JC, Lednicky JA, Wen TS, Jamieson DJ. Coronavirus Disease 2019 (COVID-19) and pregnancy: what obstetricians need to know. Am J Obstet Gynecol . 2020, 222(5):415-426

2. Meslin P, Guiomard C, Chouakria M, Porher J, Duquesne F, Tiprez C, and Zemouri N (2020). Coronavirus disease in newborns and very young infants. The Pediatric Infectious Disease Journal. 12 May 2020, 39(7): e145-e147

3. Ana Cristina Simões e Silva and Caio Ribeiro Vieira LealIs SARS-CoV-2 Vertically Transmitted? Front. Pediatr.,15 May 2020

4. Wang S, Guo L, Chen L, Liu W, Cao Y, Zhang J, et al. A case report of neonatal COVID-19 infection in China. Clin Infect Dis . 2020 ciaa225.

5. Huaping Zhu, Lin Wang, Chengzhi Fang, Sicong Peng, Lianhong Zhang, Guiping Chang, Shiwen Xia, and Wenhao Zhou. Clinical analysis of 10 neonates born to mothers with 2019-nCoV pneumonia. Transl Pediatr. 2020 Feb; 9(1): 51-60.

6.Lingkong Zeng, $\mathrm{MD}^{1}$; Shiwen Xia, $\mathrm{MD}^{2}$; Wenhao Yuan, $\mathrm{MD}^{1}$;alKai Yan, MD ${ }^{3}$; Feifan Xiao, MS ${ }^{3}$; Jianbo Shao, MD ${ }^{4}$; Wenhao Zhou, MD ${ }^{3}$ Neonatal Early-Onset Infection With SARS-CoV-2 in 33 Neonates Born to Mothers With COVID-19 in Wuhan, China JAMA Pediatr. 2020;174(7):722-725

7. Dong L, Tian J, He S, Zhu C, Wang J, Liu C, et al. Possible vertical transmission of SARS CoV-2 from an infected mother to her newborn. JAMA. 2020;323(18):1846-1848.

8.Patanè L, Morotti D, Giunta MR, et al. Vertical transmission of COVID-19: SARS-CoV-2 RNA on the fetal side of the placenta in pregnancies with COVID-19 positive mothers and neonates at birth. Am J Obstet Gynecol MFM 2020:100145. 
9.Carosso A, Cosma S, Borella F, et al. Pre-labor anorectal swab for SARS-CoV-2 in COVID-19 pregnant patients: is it time to think about it? Eur J Obstet Gynecol Reprod Biol 2020; 249:989.

10.Wang W, Xu Y, GaoR, et al. Detection of SARS-CoV-2 in different types of clinical specimens. JAMA . March 11, 2020. doi:10.1001/jama.2020.3786 